\title{
Correction to: Joint Motion Compensation and MC-Reused Spatial Error Concealment on Hardware Architecture Design
}

\section{Yu-Hsuan Lee ${ }^{1} \cdot$ Bo-Siang Huang ${ }^{1}$}

Published online: 18 February 2020

(C) Springer Science+Business Media, LLC, part of Springer Nature 2020

Correction to: J Sign Process Syst (2017) 89:319-333. https://doi.org/10.1007/s11265-016-1196-4

I am the author of this paper published in Journal of Signal processing Systems:

Yu-Hsuan Lee and Bo-Siang Huang, "Joint Motion Compensation and MC-Reused Spatial Error Concealment on Hardware Architecture Design," Journal of Signal Processing Systems, vol. 89, no. 2, pp. 319-333, Nov. 2017. (SCI, Full paper).

As a Taiwan citizen, I need to update the affiliation of the above paper, where "People's Republic of China" should be replaced by "Republic of China".

Correct affiliation should read:

Electrical Engineering/Innovation Center for Big Data and Digital Convergence, Yuan-Ze University, 135 Yuan-Tung Road, Chung-Li, 32,003, Taoyuan, Taiwan, Republic of China.

The online version of the original article can be found at https:/doi.org/ $10.1007 / \mathrm{s} 11265-016-1196-4$

Yu-Hsuan Lee

yhlee@saturn.yzu.edu.tw

Bo-Siang Huang

sfwcyihl@yahoo.com.tw

1 Electrical Engineering/Innovation Center for Big Data and Digital Convergence, Yuan-Ze University, 135 Yuan-Tung Road, Chung-Li, 32003 Taoyuan, Taiwan, Republic of China 